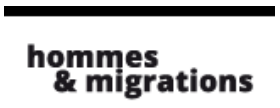

\section{Hommes \& migrations}

Revue française de référence sur les dynamiques

migratoires

\section{$1283 \mid 2010$}

Cuisines et dépendances

\title{
Le chiffon rouge
}

\section{Mustapha Harzoune}

\section{OpenEdition \\ Journals}

Édition électronique

URL : http://journals.openedition.org/hommesmigrations/1017

DOI : 10.4000/hommesmigrations. 1017

ISSN : 2262-3353

\section{Éditeur}

Musée national de l'histoire de l'immigration

\section{Édition imprimée}

Date de publication : 1 janvier 2010

Pagination : 180-188

ISBN : 978-2-919040-04-9

ISSN : $1142-852 X$

\section{Référence électronique}

Mustapha Harzoune, «Le chiffon rouge », Hommes \& migrations [En ligne], 1283 | 2010, mis en ligne le 29 mai 2013, consulté le 15 septembre 2020. URL : http://journals.openedition.org/

hommesmigrations/1017

Ce document a été généré automatiquement le 15 septembre 2020.

Tous droits réservés 


\title{
Le chiffon rouge
}

\author{
Mustapha Harzoune
}

1 Fidélité aux origines pour les uns, respect des vivants et de leur devenir pour les autres. Mais voilà, les lignes de partage ne sont pas aussi tranchées. Chacun met de l'eau dans son vin, de messe ou républicain. Alors l'origine s'acoquine avec sa descendance, futelle venue d'ailleurs. Les visionnaires des temps nouveaux ne négligent jamais de regarder dans le rétroviseur. On entend préparer l'avenir sans forligner comme on veut honorer son héritage sans insulter les vivants. Il en est de l'identité, comme de la muscade : tout serait une question de dosage.

De deux choses l'une : ou bien nous nageons en pleine politique politicienne et l'on peut dire tout et son contraire, ou bien cela traduit la complexité d'un débat dont les conclusions ne sont pas réductibles à un décret gouvernemental. L'identité se refuse à être enrégimentée. Comme le rappelle Souleymane Bachir Diagne, professeur à l'université de Columbia, dans Le Quoditien du Sénégal, le 31 décembre, citant le philosophe Wittgenstein, cette question de l'identité est "le diable en personne". Cela ne rassurera pas les inquiets.

\section{Pour qui sonne le “La”...}

3 Car, à en croire M. Guaino, a France serait inquiète. La France? Pas seulement. Le 8 décembre il déclare sur Europe 1: "Il y a une sourde inquiétude dans les populations européennes, celle de voir dénaturés leur façon de vivre, leur mode de relations sociale, leur mode de pensée, leur mode de croyance." Et à propos d'on ne sait quel "défi" : "Ceux qui viennent doivent toujours éviter de lancer un défi à ceux qui sont là, dans leurs croyances et leurs convictions." Quant aux musulmans vivant en France depuis "une, deux et parfois trois générations", "ils ont à être accueillis dans une autre civilisation et une autre culture". Eh oui... trois générations plus tard, on est encore et toujours "accueilli".

Le 14, il persiste : “[...] Dire que l'immigration ou la question religieuse n'ont rien à voir avec la crise identitaire qui mine [sic] toute l'Europe serait absurde." Et de poser ces questions: "Comment l'islam peut-il s'adapter à la laïité, à la séparation du spirituel et du temporel ou à l'égalité de l'homme et de la femme? Comment allons-nous résoudre le problème qui nous est 
posé par des jeunes nés en France qui [...] définissent leur identité par opposition à l'identité française? Comment allons-nous surmonter la tension de plus en plus forte qui se fait jour entre les tentations communautaristes et notre modèle de République 'une et indivisible?"

Ces mises en garde viseraient à préparer l'avenir : "Si l'on refuse le communautarisme, le développement séparé, si l'on veut vivre ensemble, il faut accepter le mélange, le métissage. Pour que cela soit possible, il faut exclure toute attitude de défi, de provocation, d'ostentation. [...] Une assimilation réussie, c'est la clé du métissage."

De la peur à la mise en cause, de la dénonciation à la mise en garde, de l'avertissement à la mise au pas..., la pente est savonneuse. Le débat sur l'identité nationale est "nécessaire ", dixit N. Sarkozy, le 12 novembre à La Chapelle-en-Vercors. Après avoir évoqué l'“ abandon", la "haine de soi", la "haine des siens" et la "détestation de son propre pays", il prévient: "La France est un pays de tolérance et de respect, mais elle demande aussi qu'on la respecte." Et qu'est ce qui ne respecterait pas la France ? La burqa. Mais aussi ceux qui entendent "bénéficier de la Sécurité sociale sans jamais se demander ce que l'on peut faire pour son pays"; ceux qui veulent "bénéficier des allocations chômage sans se sentir moralement obligés de tout faire pour retrouver du travail" ; ceux qui voudraient "profiter de la gratuité des études [...] et ne pas être assidus aux cours, ne pas témoigner de la considération pour leurs professeurs, ne pas respecter les bâtiments qui les accueillent".

7 Et le 9 décembre, après la votation suisse sur les minarets, le président se fend d'une tribune dans Le Monde qui fleure bon la plume de Guaino: "Cette sourde menace que tant de gens dans nos vieilles nations européennes sentent, à tort ou à raison, peser sur leur identité, nous devons en parler tous ensemble de peur qu'à force d'être refoulé ce sentiment ne finisse par nourrir une terrible rancœur." Là encore, tout cela débouche sur un éloge du "changement ", de "l'ouverture aux autres" et du "métissage".

8 Le métissage version Sarkozy-Guaino, c'est un peu la Bibliothèque rose : “C'est de la part de celui qui accueille l'offre de partager son héritage, son histoire, sa civilisation, son art de vivre. C'est de la part de celui qui arrive la volonté de s'inscrire sans brutalité [sic], comme naturellement [resic], dans cette société qu'il va contribuer à transformer, dans cette histoire qu'il va désormais contribuer à écrire. La clé de cet enrichissement mutuel qu'est le métissage des idées, des pensées, des cultures, c'est une assimilation réussie."

9 Le président de tous les Français apostrophe "ses compatriotes musulmans" pour les mettre en garde : "Je veux leur dire aussi que, dans notre pays, où la civilisation chrétienne a laissé une trace aussi profonde, où les valeurs de la République sont partie intégrante de notre identité nationale, tout ce qui pourrait apparaître comme un défi lancé à cet héritage et à ces valeurs condamnerait à l'échec l'instauration si nécessaire d'un islam de France."

Déjà à La-Chapelle-en-Vercors, le président affirmait : "Il n'y a pas un libre penseur qui ne se sente au fond de lui héritier de la chrétienté, qui a laissé tant de trace." Et d'ajouter pourtant : "On est Français parce qu'on ne se reconnaît pas dans une race, qu'on ne se laisse pas enfermer dans une origine ou dans une religion."

11 À propos de la tribune du Monde, Dounia Bouzar relève : "Sarkozy traite dans le premier paragraphe de l'immigration, en faisant le distinguo entre 'accueillant' et 'accueilli', et dans le second de l'islam. Or, ça fait vingt ans que l'on se bat pour séparer la question de l'immigration de celle de l'islam. La deuxième idée grave, c'est de présenter les civilisations comme des blocs monolithiques sans interactions entre les uns et les autres. Cela relève du mythe. Sarkozy parle comme s'il y avait un monde bipolaire avec d'un côté l'occident, sa civilisation, sa culture, son art de vivre, et de l'autre des étrangers, porteurs d'aucune valeur, qui doivent s'inscrire dans 
l'histoire de France sans la brutaliser. Cela me fait penser aux discours des islamistes qui veulent faire croire que toutes les valeurs sont intrinsèquement dans l'islam, qui a tout inventé, la civilisation, le féminisme, la laïcité."(Libération, 9 décembre)

\section{Dérapages (in)contrôlés}

12 Ce fut bien d'en haut que le chiffon rouge de la "peur", de la "sourde inquiétude", de la " sourde menace", du "défi" fut d'abord agité. Certains Français n'ont fait que relayer ce qu'ils ont pu lire ou entendre dans leur médias ou dans le kit des débats - une liste de thèmes et 200 questions - concocté par le ministère. Pour L'Humanité ce "Guide pratique antirépublicain" est "un questionnaire aux allures de pamphlet antirépublicain qui présente les immigrés comme faisant courir des risques pour la France" (le 23 novembre). À ce propos, l'anthropologue Aïssatou Mbodj-Pouye note la disparition d'une curieuse question : “ Comment éviter l'arrivée sur notre territoire d'étrangers en situation irrégulière, aux conditions de vie précaires génératrices de désordres divers (travail clandestin, délinquance) et entretenant, dans une partie de la population, la suspicion vis-à-vis de l'ensemble des étrangers ?" (Libération , 4 décembre)

13 Eric Besson n'a pas caché un autre motif à ce débat, électoraliste celui-là : la peur du FN ! Et quoi de plus simple que d'aller marauder sur les terres de la famille Le Pen pour lui "piquer définitivement un certain nombre de valeurs qu'il croyait s'être attribuées et qui ne sont pas ses valeurs. Il n'y a aucune raison de lui laisser Marianne, la République, le drapeau, l'hymne national." (La Croix.fr, $1^{\mathrm{er}}$ novembre) D'ailleurs, selon Brice Hortefeux, "le message" [de N. Sarkozy] était : "Affirmez vos convictions, n’hésitez pas à cliver, les Français nous soutiennent", rapporte Le Monde du 3 décembre.

Mais voilà, une semaine après avoir constaté les dérapages, on craint "l'effet boomerang ", c'est-à-dire un coup de bâton porté à l'UMP par... un FN ragaillardi. Le 27 novembre à Bailleul (Nord), où un lieu d'accueil pour les migrants allait s'ouvrir deux jours après sa conférence de presse, Marine Le Pen servait autre chose que du Canada Dry: "Nous sommes dans le symbole de ce que nous dénoncions, le cancer d'une immigration clandestine qui se métastase à l'ensemble du département, puis de la région et demain à l'ensemble de la France."

15 Ces dérapages, celui d'André Valentin, maire de Gussainville (Meuse) et les sorties de Nadine Morano ou de Pascal Clément, ont fini par inquiéter les rangs de la droite. "De Villepin à Juppé en passant par Raffarin ou Yazid Sabeg, de plus en plus de voix dans la majorité s'inquiètent des dérives du débat lancé par Eric Besson." (Le Figaro, 16 décembre) Et les appels à "refuser" le débat (Médiapart le 2 novembre et Le Monde du 24 novembre), à “ supprimer" le ministère de l'Identité nationale (Libération, 4 décembre), à "arrêter ce débat" (Libération, 21 décembre) ou en faveur "d'un autre débat" (Le Monde, 29 décembre) se sont multipliés.

16 Le 4 décembre, Laurent Joffrin résume la situation: "Et si le grand débat bessonien sur l'identité nationale n'était finalement qu'un piège à c... dans lequel l'UMP est tombée après l'avoir tendu ? [...] Les initiateurs du débat voulaient user de la peur pour accroître leurs soutiens. Ils s'aperçoivent un peu tard qu'ils ont ouvert une boite de Pandore." (Libération)

17 Pour Dominique Lefebvre, maire PS de Cergy, Nicolas Sarkozy est un "pompier pyromane": "Vous avez beau parler de 'métissage' ou d"ouverture', vous utilisez ces termes comme des gadgets, qui révèlent plus qu'ils ne masquent l'équation simpliste qui sous-tend tout 
votre propos $:$ identité nationale $=$ racines chrétiennes $;$ immigration $=$ islam $;$ islam $=$ risque de 'dénaturation' pour l'identité française. Nous sommes là dans le registre du pur et de l'impur. Voilà pour ceux qui auraient pris au sérieux votre passage sur la nécessaire évolution au contact des autres." (Le Monde, 11 décembre)

\section{Intégration ou stigmatisation}

18 Pour le quotidien La Croix, "les concurrences identitaires à l'œuvre dans la société moderne soulèvent des questions inédites" et "de nombreux intellectuels s'inquiètent d'une rupture irrémédiable des jeunes générations avec la langue française et la culture classique" ( 1 er novembre). Plus largement, le linguiste Alain Bentolila demande: "Comment peut-on parler d'identité nationale sans prendre l'engagement que le pouvoir linguistique soit équitablement distribué sur notre territoire? Les inégalités linguistiques que l'on tolère parfois avec complaisance défont notre tissu social et encouragent les communautarismes."

19 “Défendre l'identité nationale c'est d'abord mener le combat pour permettre à chaque citoyen de transmettre à l'autre sa pensée de la façon la plus juste et la plus précise, et d'ouvrir en retour son intelligence à la pensée de l'autre avec discernement." (Le Monde, 11 novembre)

Pour la sociologue Claudine Attias-Donfut, "on occulte la réalité de l'intégration [...]. Il serait plus utile de débattre de notre modèle d'intégration. À mon sens, il faut tourner le dos à l'idée d'assimilation des étrangers, qui nie les différences et l'identité de chaque personne dans son appartenance. Il faut conserver l'esprit de l'intégration à la française en reconnaissant l'existence d'identités multiples." (Le Monde, 4 décembre)

21 À propos de ce modèle "largement imaginaire”, l'historienne Esther Benbassa, écrit qu'il " $n$ 'a aucune chance de fonctionner en nos temps de fortes revendications identitaires et/ou communautaires. [...] Qu'on les approuve ou pas, ces revendications, nos gouvernants ne pourront pas en faire indéfiniment fi. De surcroît, pourquoi ne serait-on pas à la fois un bon musulman et un bon Français, comme on est à la fois femme et française ? [...] La France se targue d'avoir "assimilé" les Juifs au XIX siècle. Les historiens savent pourtant qu'ils ne se sont pas "assimilés", mais acculturés, sachant, pour nombre d'entre eux, conjuguer les valeurs de la République avec celles du judaïsme." (Rue89.com, 3 novembre)

"Et si l'intégration réussissait?" demande Alain Duhamel dans Libération du 10 décembre. “À la longue, à travers les décennies et les siècles, l'intégration a toujours réussi. [...] La situation actuelle n'est pas pire, tant s'en faut, et ne tient évidemment pas à l'islam. La grande majorité des musulmans s'intègrent lentement mais pas plus lentement que les Polonais venus sous la conduite de leurs prêtres. [...] À côté de ce mouvement historique, la querelle des minarets est absurde et celle des mosquées, artificielle."

Soulevant "la question du rapport à l'histoire", Max Gallo se veut plus circonspect : "La réalité sociale et économique ne favorise pas l'intégration : il n'y a plus d'école ni de service militaire... Nous n'avons pas dit aux immigrés que nous sommes un pays qui a déjà une histoire dans laquelle ils doivent s'insérer pour la modifier, comme nous l'avions fait sous la III République avec les immigrés italiens, polonais. Ce n'est pas parce qu'ils étaient Européens que c'était plus facile. Il ne s'agit pas de dire 'nous sommes des Gaulois', mais 'il y a des Gaulois' [...]." Et de préciser : "La France s'est construite dans un amalgame permanent entre des individus arrivant de l'étranger et puis ce sol, ce lieu, cette histoire de France. [...] Autrement dit, nous sommes ouverts. [La France] est le pays d'une histoire, de l'étranger dans chaque Français." (Le Point.fr, 2 décembre) 

financier français. Ils se prennent pour des Blacks Panthers américains qui luttent contre l'esclavage. Ils dealent, ils volent, ils braquent, ils brûlent, ils agressent en jurant à tour de bras. Tout cela au nom de ce que l'on a fait à leurs parents. Leur icône : Tony Montana. Scarface. Superproduction américaine. Leur musique : 50 Cent, Eminem, Marvin Gaye... Leur resto préféré : le McDo. Tu parles de rebelles, de terroristes... ils sont labélisés depuis leur caleçon jusqu'à leur ceinturon. Ils sont américains." Il demande "le droit à l'anonymat', autrement dit 'qu'on lui foute la paix!"' "Le pire, c'est que cette manière de concevoir ma place dans la société n'appartient pas seulement à ceux qu'on pourrait appeler 'racistes'. Non, je vous parle de l'ambiance générale. Les bobos, les babas et les bling-bling ont tous dans leur caboche une ou deux petites blagues, deux ou trois craintes sur ces chers Français... d'origine maghrébine."

\section{Fierté !}

Il serait aussi "nécessaire" de restaurer confiance et fierté nationales. Être Français, " c'est être fier de vivre en France", déclare Xavier Bertrand (Nouvelobs.com, $1^{\mathrm{er}}$ décembre).

"Il était temps!" écrit Ivan Rioufol, le 6 novembre sur son blog. Le chroniqueur du Figaro conseille au gouvernement de chausser les bottes du FN: "Un réveil des consciences populaires prend forme, que le gouvernement se devra d'accompagner s'il ne veut pas voir l'extrême droite canaliser les frustrations qui surgissent. L'erreur serait pour lui de s'enfermer dans l'idéologie ambiguë du 'métissage impératif [...]." "Il n'est certes pas interdit de s'interroger sur la légèreté des élites qui persistent à laisser s'installer des peuples étrangers à l'esprit occidental sans se soucier de leur intégration. Mais ceux-là ne sont pour rien dans le mal identitaire né d'un désintérêt de soi et d'un attrait pour l'uniformisation par le bas." 
uloir réduire la France aux droits de l'homme, à son modèle social et à ses services publics [...] revient à entériner l'effacement progressif d'un peuple et à refuser à chaque civilisation le besoin vital, que leur reconnaissait Claude Lévi-Strauss qui vient de mourir, de se distinguer des autres."

pe Descola, professeur au Collège de France, parle justement de Lévi-Strauss : Toute son expérience d'ethnologue montre que l'identité se forge par des interactions sur les frontières, sur les marges d'une collectivité. L'identité ne se constitue en aucune façon d'un catalogue de traits muséifiés, comme c'est souvent le cas lorsque des États s'emparent de la question de l'identité nationale. Les sociétés se construisent une identité, non pas en puisant dans un fonds comme si on ouvrait des boîtes, des malles et des vieux trésors accumulés et vénérés, mais à travers un rapport constant d'interlocution et de différenciation avec ses voisins. Et c'est cette double expérience, personnelle et politique d'un côté et d'ethnologue de l'autre, qui l'a conduit à récuser et vivement critiquer l'accaparement, par des États, de l'identité nationale." (Le Monde.fr, 4 novembre)

Maurice T. Maschino, après avoir cité Montesquieu : "Je suis nécessairement homme et [...] je ne suis Français que par hasard", affirme qu'"il n'y a pas à être 'fier' de ce hasard, qui, par définition, est totalement gratuit. En faire une valeur et l'objet d'un débat national est une escroquerie" (Le Monde, 30 décembre). Souleymane Bachir Diagne écrit: "Comme s'il y avait pour l'humain, ainsi que le faisait remarquer le philosophe cynique [Wittgenstein], le moindre mérite à être du terroir, puisqu'il s'agit alors d'un privilège que l'on partage avec les escargots et les limaçons." (Le Quotidien du Sénégal, 31 décembre)

\section{Les "vrais" Français et les autres}

Ce débat peut-il dresser une partie des Français contre une autre ? C'est ce que pense le sociologue Michel Wieviorka (La Croix.fr, $1^{\mathrm{er}}$ novembre) ou l'anthropoloque Régis Meyran pour qui "parler de l'identité nationale revient au même que de parler de 'Français de souche', ça renvoie à une opposition entre les supposés vrais Français et les étrangers" (L'Express.fr, 2 novembre).

Dans une note du 4 novembre pour la Fondation Terra Nova (proche du PS), le philosophe Joël Roman écrit: 'En faisant mine de s'inquiéter d'une 'identité nationale' qui n'est ni contestée, ni menacée, le gouvernement de Nicolas Sarkozy prend un risque considérable: celui de conforter les peurs, d'attiser les inquiétudes, de figer cette fameuse 'identité nationale' autour de critères de comportements ou d'habitudes majoritaires, bref, met en péril cette cohésion même qu'elle prétend conforter. Elle va dégénérer en repli nationaliste, car il faudra des boucs émissaires. Ce sera l'Europe, ce sera l'immigration : pas même seulement l'immigration des nouveaux venus, mais celle des plus anciennement arrivés, cette immigration que plusieurs générations de nationalité française n'ont pas pu effacer car elle porte les stigmates de l'étranger : couleur de peau, langue parfois, religion."

Dans Le Monde du 27 décembre, Emmanuel Todd qualifie ce débat de "pervers" : "Ce que Sarkozy propose, c'est la haine de l'autre." "Le gouvernement, à l'approche d'une échéance électorale, propose, je dirais même impose, une thématique de la nation contre l'islam. Je suis révulsé comme citoyen. En tant qu'historien, j'observe comment cette thématique de l'identité nationale a été activée par en haut, comme un projet assez cynique." Le démographe estime que "la réalité de la France est qu'elle est en train de réussir son processus d'intégration. Les populations d'origine musulmane de France sont globalement les plus laïcisées et les plus

Hommes \& migrations, 1283 | 2010 
intégrées d'Europe, grâce à un taux élevé de mariages mixtes". "Le tempérament égalitaire des Français fait qu'ils n'en ont rien à foutre des questions de couleur et d'origine ethnique ou religieuse!"

"Quand on est confronté à un pouvoir qui active les tensions entre les catégories de citoyens français, on est quand même forcé de penser à la recherche de boucs émissaires telle qu'elle a été pratiquée avant-guerre." Sur cette question, Edwy Plenel, dans "Une honte nationale" mis en ligne le 18 décembre sur Médiapart, a donné la plus serrée des critiques des discours de Nicolas Sarkozy à La Chapelle-en-Vercors et de François Fillon à l'Institut Montaigne à Paris le 4 décembre. Jean-François Bayart voit aussi “dans la démarche d'Eric Besson et de Nicolas Sarkozy une espèce d'accaparement de la complexité de l'histoire française qui rappelle de biens mauvais souvenirs du point de vue de notre histoire" (Le Monde, 7 novembre).

Enfin, dans son éditorial du 17 décembre, Le Monde écrit : "Le rôle et la responsabilité du président sont de faire en sorte que la République rassemble les Français plutôt que les dresser les uns contre les autres, intègre au lieu d'exclure, se nourrisse des différences plutôt que les exacerber, réduise les inégalités plutôt que les creuser. Nicolas Sarkozy s'honorerait donc d'admettre son erreur. Et de la corriger."

\section{Le dialogue de l'un et du multiple}

Dans Libération du 16 décembre, la sociologue Maryse Tripier rapporte que "la notion d'identité est très discutée dans les sciences sociales parce qu'elle fige. Or tout le monde a des identités multiples, professionnelles, culturelles, d'âge, territoriales. Il y a 365 manières d'être musulman, ou catholique, ou juif. Dans les enquêtes que nous faisons, personne ne demande à être identifié par sa race, son origine ou sa religion, mais par son diplôme ou son action."

Pap N'Diaye, de l'École des hautes études en sciences sociales, craint une dérive “ autoritariste" du débat: "Il y a tellement de façons d'être Français qu'il serait triste que le gouvernement nous dicte ce qu'est être Français." (Le Monde, 3 novembre)

Même idée chez Esther Benbassa: "Une identité qui n'est évidemment pas seulement nationale, mais pluriculturelle, fluctuante et de plus en plus européenne". "Il y a certes un débat à mener sur l'identité comme telle d'abord, ses transformations, ses reconstructions, ses ambiguïtés, sur celle de la France réelle, ensuite, largement métissée et hétérogène. Ce débat intégrerait indéniablement la question de ces identités individuelles multiples et mouvantes qui sont les nôtres [...]." (Rue89.com, 3 novembre)

Joël Roman note que "c'est face aux autres, reconnu ou au contraire nié par eux, que j'éprouve mon identité. Et ces identités sont multiples, en fonction des situations où nous nous trouvons, des interlocuteurs à qui l'on a affaire. C'est pourquoi l'identité nationale n'est qu'une composante parmi d'autres des identités politiques et culturelles qui sont les nôtres [...]. La règle ici est la suivante : n'assigner jamais aucune identité à l'autre, mais lui permettre de déployer son identité dans les termes qu'il souhaite." (Fondation Terra Nova, 4 novembre)

\section{La politique et le marché}

Pour Jacques Reland du Global Policy Institute, à Londres : "Le modèle social français fait partie de l'identité nationale française. [...] C'est Nicolas Sarkozy qui a été la plus grande menace pour l'identité quand il a parlé des défauts du modèle social français. La crise a montré la valeur de ce système. [...] En France, être Français, c'est croire que le politique l'emporte sur le marché." 
“Ce qui est remarquable chez les Français, c'est que chacun se sent aussi digne que l'autre. [...] La fragilité de l'ascenseur social est, à cet égard, une grande menace." (Libération, 3 novembre)

À propos du "désir de vivre ensemble", Le Monde cite l'historien Vincent Duclert, l'auteur de La France, une identité démocratique (Seuil): "Dans une République qui n'avait pas véritablement de Constitution, ce sont les grandes lois qui ont servi de textes fondateurs : sur la liberté de la presse (1881), sur l'école (1881-1882), sur les syndicats (1884), sur la liberté d'association (1901), sur la séparation des Églises et de l'État (1905)...' L'existence de ce 'patrimoine commun de droits et de libertés', dont la liste est par définition ouverte, constitue ce que Vincent Duclert appelle donc l'identité démocratique de la France - expression qu'il préfère à celle d'identité nationale, dans la mesure où elle met l'accent sur un 'projet politique' en devenir plutôt que sur une 'définition essentialiste' fixée une fois pour toutes." (7 novembre)

D'ailleurs, pour L'Humanité du 20 novembre, “l'identité nationale' version Sarkozy a de quoi combler Laurence Parisot, la patronne des patrons. [...] Il s'agit aujourd'hui, de sortir de 1945 et de défaire méthodiquement le programme du Conseil national de la Résistance! C'est-à-dire la sécurité sociale, les retraites par répartition, les services publics, la protection judiciaire de la jeunesse, et tout cet inventaire social à la Prévert, toutes ces vieilleries qui empêchent la France de saisir les merveilleuses opportunités offertes par la mondialisation capitaliste. Place à la 'modernité. La France de la casse des droits sociaux et des protections collectives, 'étranger' ou pas, tu l'aimes ou tu la quittes!"

Pour Mohamed Mechmache, du collectif AC-Lefeu né après les émeutes urbaines de 2005, "le vrai débat doit porter sur la lutte contre l'exclusion dont se sentent victimes les jeunes de banlieue. Ce qu'ils réclament pour se sentir pleinement Français, c'est du droit commun, l'accès aux services publics, à un travail" (La Croix.fr $1^{\mathrm{er}}$ novembre). Même idée développée par Gérard Collomb, le maire de Lyon, dans Libération le 4 novembre.

Bénédicte Charles, dans Marianne du 3 novembre, demande alors: “À quoi sert ce débat dont les conclusions, en forme de concept creux, de slogan publicitaire, sont déjà connues? Diver-si-té ! On n'a pas fini de l'entendre, ce mot - et donc, par raccroc, la justification des inégalités, puisque ce n'est pas parce que la société est multiculturelle qu'elle est plus égalitaire. Alors, à quoi sert le débat sur l'identité nationale? À rien, sinon à clore le débat. Pour longtemps.

\section{RÉSUMÉS}

À lire les contributions parues dans la presse, une question se pose : n'y aurait-il rien de nouveau sous le soleil identitaire depuis Barrès et Renan ? Ceux que l'on pourrait rattacher au premier souvent à leur corps défendant - ressortent d'une vieille boîte, la terre, la religion, chrétienne bien sûr, le sang, des ancêtres et des morts, une langue, etc. "Ce que l'ethnologue Orvar Löfgren a appelé fort justement un 'kit' identitaire." (Le Monde, 7 novembre) Les autres offrent de l'identité une définition "à la Renan" : volontaire, évolutive, syncrétique, parfois contradictoire, oublieuse certainement et même faillible. 